This document is confidential and is proprietary to the American Chemical Society and its authors. Do not copy or disclose without written permission. If you have received this item in error, notify the sender and delete all copies.

\title{
Carrier Extraction from Perovskite to Polymeric Charge Transport Layers Probed by Ultrafast Transient Absorption Spectroscopy
}

\begin{tabular}{|r|l|}
\hline Journal: & The Journal of Physical Chemistry Letters \\
\hline Manuscript ID & jz-2019-02502r.R1 \\
\hline Manuscript Type: & Letter \\
\hline Author: & nubmitted by the \\
\hline Complete List of Authors: & $\begin{array}{l}\text { Ugur, Esma; King Abdullah University of Science and Technology } \\
\text { Physical Sciences and Engineering Division, Material Science and } \\
\text { Engineering } \\
\text { Khan, Jafar; King Abdullah University of Science and Technology, } \\
\text { Physical Sciences and Engineering Division } \\
\text { Aydin, Erkan; King Abdullah University of Science and Technology } \\
\text { Physical Sciences and Engineering Division, Materials Science and } \\
\text { Engineering } \\
\text { Wang, Mingcong; King Abdullah University of Science and Technology } \\
\text { Kirkus, Mindaugas; Imperial College London, Chemistry } \\
\text { Neophytou, Marios; King Abdullah University of Science and Technology } \\
\text { McCulloch, Iain; Imperial College London, Chemistry } \\
\text { De Wolf, Stefaan; King Abdullah University of Science and Technology, } \\
\text { Laquai, Frédéric; King Abdullah University of Science and Technology, } \\
\text { Physical Sciences and Engineering }\end{array}$ \\
\hline
\end{tabular}

\section{SCHOLARONE" Manuscripts}




\title{
Carrier Extraction from Perovskite to Polymeric
}

\author{
Charge Transport Layers Probed by Ultrafast
}

\section{Transient Absorption Spectroscopy}

\author{
Esma Ugur ${ }^{\dagger}$, Jafar I. Khan ${ }^{\dagger}$, Erkan Aydin, Mingcong Wang, Mindaugas Kirkus, Marios \\ Neophytou, Iain McCulloch, Stefaan De Wolf, Frédéric Laquai* \\ King Abdullah University of Science and Technology (KAUST), KAUST Solar Center (KSC), \\ Physical Sciences and Engineering Division (PSE), Thuwal, 23955-6900, Kingdom of Saudi \\ Arabia
}

\section{Corresponding Author \\ * Frédéric Laquai}

Contact information: frederic.laquai@kaust.edu.sa 


\begin{abstract}
The efficiency of state-of-the-art perovskite solar cells is limited by carrier recombination at defects and interfaces. Thus, understanding these losses and how to reduce them is the way forward towards the Shockley-Queisser limit. Here, we demonstrate that ultrafast transient absorption spectroscopy can directly probe hole extraction, and recombination dynamics at perovskite/hole transport layers (HTLs) interfaces. To illustrate this, we employed PDPP-3T as HTL, since its ground state absorption is at lower energy than the perovskite's photobleach, enabling direct monitoring of interfacial hole extraction and recombination. Moreover, by fitting the carrier dynamics using diffusion model, we determined the carrier mobility. Then, by varying the perovskite thickness, we distinguished between carrier diffusion and carrier extraction at the interface. Lastly, we prepared device-like structures, $\mathrm{TiO}_{2} /$ perovskite/PDPP-3T stacks, and observed reduced carrier recombination in the perovskite. From PDPP-3T carrier dynamics, we deduced that hole extraction is one-order faster than recombination of holes at the interface.
\end{abstract}

TOC GRAPHICS

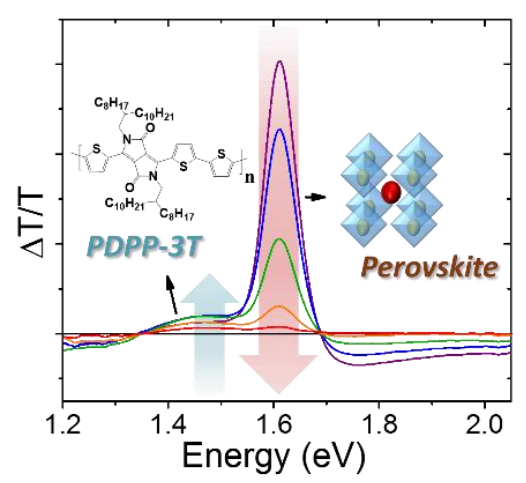

KEYWORDS perovskite solar cells, ultrafast spectroscopy, interface recombination, hole extraction, carrier dynamics 
Perovskite solar cells (PSCs) have shown remarkable progress in power conversion efficiency (PCE) - nowadays achieving values above $25 \%^{1}$ - making them one of the most promising photovoltaic technologies combining high performance with low fabrication costs. This rapid progress has been possible because of the outstanding optical and electronic properties of metal halide perovskites such as their sharp optical absorption onset and steep absorption edge (suggesting low Urbach energies), balanced electron-hole diffusion length, and high defecttolerance for a solution-processed material..$^{2-9}$

To unlock the full performance potential of PSCs, the choice of electron and hole transport layers (ETL and HTL) plays a key role, since these layers govern charge extraction and transport, but also, to some extent, interface recombination. ${ }^{10-14}$ Efficient charge extraction and low interface recombination are required to achieve high internal quantum efficiency (IQE), an important criterion for device quality. ${ }^{15}$ Understanding the charge-extraction and recombination mechanisms at the respective interfaces is of significant importance for further progress in device performance. To date, the best PSCs are of the so-called $n-i-p$ configuration, employing either mesoporous titanium dioxide $\left(\mathrm{m}-\mathrm{TiO}_{2}\right)$ or nanocrystalline tin oxide $\left(\mathrm{SnO}_{2}\right)$ layers as ETLs. ${ }^{13-14,16}$ As HTLs, a large variety of inorganic and organic materials such as nickel oxide $\left(\mathrm{NiO}_{\mathrm{x}}\right), 2,2^{\prime}, 7,7^{\prime}-$ Tetrakis[N,N-di(4-methoxyphenyl)amino]-9,9'-spirobifluorene (spiro-OMeTAD), poly(triarylamine) polymer (PTAA), donor-acceptor type materials, conjugated small-molecules, porphyrin dimers, and diketopyrrolopyrrole (DPP) based polymers have already been proposed and tested. ${ }^{12-13,17-26}$

Charge extraction and recombination processes have been studied intensively using different transient spectroscopy techniques such as transient photocurrent, microwave conductivity, and absolute photoluminescence (PL) intensity as well as time-resolved PL spectroscopy. ${ }^{15}{ }^{27-29} \mathrm{In}$ 
principle, the hole extraction process at the perovskite/HTL interface can be probed by transient absorption (TA) spectroscopy, which allows probing of the excited-state dynamics on different time scales. ${ }^{30-34}$ This is typically achieved through monitoring the evolution of the ground state bleach (GSB) of the perovskite; the associated extraction time of charge carriers is typically in the range from sub-picoseconds to sub-nanosecond. ${ }^{33-37}$ The broad timescale can be explained by variations in the perovskite crystal properties and quality of the perovskite/HTL interface. However, discriminating between hole extraction on the one hand and interfacial carrier recombination processes on the other hand is not straightforward. ${ }^{13,31}$ This is in part due to the spectral overlap of the photoinduced bands of commonly-used HTLs with the GSB of the perovskite. Here, we mitigate this challenge by employing a specific polymer, namely PDPP-3T, as HTL. Using PDPP-3T allows direct probing of hole extraction dynamics since its ground state absorption is in the near-infrared (NIR) range and thus red-shifted compared to the perovskite's GSB. To gain insight into the hole extraction dynamics, optical excitation was carried out from both the perovskite and HTL sides in our TA measurements. Moreover, we varied the thickness of the perovskite layer and observed that with increasing perovskite layer thickness, hole extraction is slowed, when exciting from the perovskite side (opposite to the HTL). Lastly, we extended our measurements to samples that mimic the $n-i-p$ solar cell architecture by sandwiching the perovskite absorber layer between $\mathrm{TiO}_{2}$ and PDPP-3T. Interestingly, we observed that charge carrier extraction at the respective transport layers results in reduced recombination, indicated by the slower recovery of the perovskite's ground state.

The perovskite absorber layers used in our study were mixed-cation lead mixed-halide perovskite $\left(\mathrm{Cs}_{0.07} \mathrm{Rb}_{0.03} \mathrm{FA}_{0.765} \mathrm{MA}_{0.135} \mathrm{PbI}_{2.55} \mathrm{Br}_{0.45}\right)$ chosen because of their excellent reproducibility, phase stability, and high photovoltaic performance. ${ }^{13}$, 38-39 Three different 
perovskite thicknesses, $170 \mathrm{~nm}, 360 \mathrm{~nm}$, and $570 \mathrm{~nm}$ were prepared by varying the concentration of perovskite precursor solution, as explained in the Experimental Section in the Supporting Information. Prior to the spectroscopic investigation, we analyzed the structural and optical properties of the perovskite film and neat PDPP-3T polymer film. Scanning electron microscopy (SEM) was used to examine the surface quality of the perovskite films and showed that increasing the film thickness improved the film quality (Figure S1). The steady-state absorption spectra of the perovskite films with different thicknesses and of the PDPP-3T polymer are presented in Figure 1a. We note that the absorption spectrum of PDPP-3T covers the range from $550 \mathrm{~nm}$ to the NIR, which is different from the absorption spectrum of the commonly-used HTL spiro-OMeTAD (Figure S2). Additionally, a marked change in absorbance was observed when the perovskite film thickness was increased from 170 to $570 \mathrm{~nm}$, while the bandgap remained virtually unchanged, $1.58 \pm 0.02$ and $1.57 \pm 0.02 \mathrm{eV}$. The difference can be explained by increased crystallite size (Figure S3). ${ }^{40}$ 
(a)

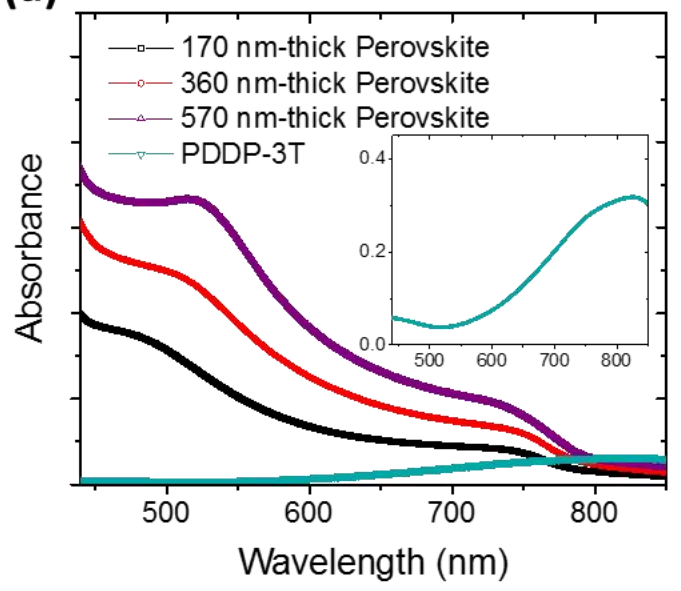

(c)

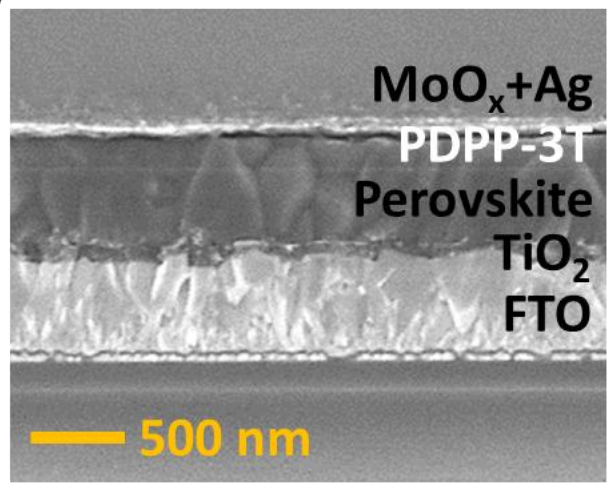

(b)

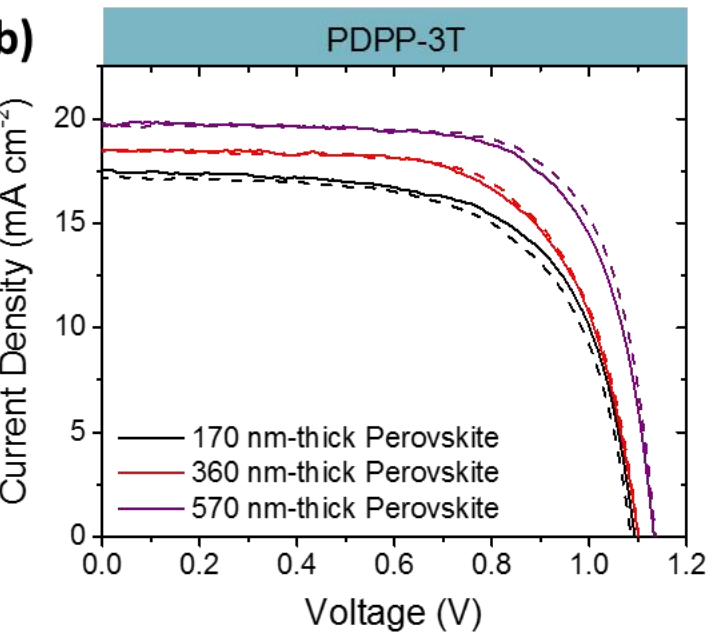

(d)

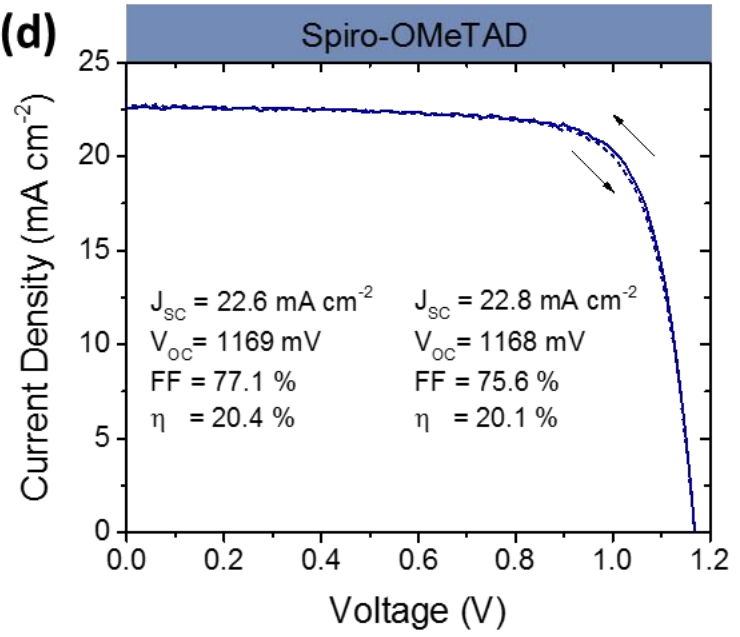

Figure 1. (a) Steady-state absorption spectra of the PDPP-3T polymer and perovskite films with different thicknesses. (b) $J-V$ characteristics of the PSCs with PDPP-3T HTL, (c) cross-sectional SEM image of the device with $570 \mathrm{~nm}$-thick absorber layer (scale bar: $500 \mathrm{~nm}$ ), and (d) $J-V$ characteristics of the reference device with spiro-OMeTAD. Straight and dashed lines represent the forward and reverse scans, respectively.

To verify that the PDPP-3T polymer effectively acts as HTL for $n-i-p$ PSCs, devices of the structure $\mathrm{FTO} / \mathrm{c}-\mathrm{TiO}_{2} / \mathrm{m}-\mathrm{TiO}_{2} / \mathrm{Cs}_{0.07} \mathrm{Rb}_{0.03} \mathrm{FA}_{0.765} \mathrm{MA}_{0.135} \mathrm{PbI}_{2.55} \mathrm{Br}_{0.45} / \mathrm{PDPP}-3 \mathrm{~T} / \mathrm{Ag}$ (Figure 1b and Table 1) were fabricated. Cross-sectional SEM was employed to determine the thicknesses of the individual layers in the stack; representative images are presented in (Figure 1c and Figure 
S4). We determined thicknesses of $500 \mathrm{~nm}$ for FTO, $100 \mathrm{~nm}$ for c- $-\mathrm{TiO}_{2} / \mathrm{m}-\mathrm{TiO}_{2}$ and $170 \mathrm{~nm}, 360$ $\mathrm{nm}$, and $570 \mathrm{~nm}$ for the different perovskite layers, $60 \mathrm{~nm}$ for PDPP-3T, and $90 \mathrm{~nm}$ for Ag. The device with the $570 \mathrm{~nm}$-thick perovskite absorber layer had the highest PCE of $16.1 \%$ (Table 1). We observed a remarkable enhancement in short-circuit current densities $\left(\mathrm{J}_{\mathrm{SC}}\right)$ from 17.5 to 19.7 $\mathrm{mA} \mathrm{cm}{ }^{-2}$ when increasing the absorber layer thickness, as expected due to the increased absorbance. Furthermore, the open-circuit voltage $\left(\mathrm{V}_{\mathrm{OC}}\right)$ of the devices was improved from 1088 to $1133 \mathrm{mV}$. The increment in $\mathrm{V}_{\mathrm{OC}}$ is either due to an enhanced perovskite crystal quality obtained in thicker films or the presence of interface recombination, which becomes less dominant for thicker perovskite films with improved quality. ${ }^{41-43}$ Consequently, the PCE of $12.5 \%$ of the 170 nm-thick perovskite device improved to $16.1 \%$ for the 'standard' device thickness $(570 \mathrm{~nm})$. The stabilized efficiencies of the champion devices with different thicknesses are shown in Figure S5; they demonstrate that devices retain their initial PCE for more than 10 minutes. The external quantum efficiency (EQE) spectra for PDPP-3T-based devices as well as the integrated current densities for perovskites of different thickness are presented in Figure S7. The integrated current density of $18.8 \mathrm{~mA} \mathrm{~cm}^{-2}$ for the regular device thickness $(570 \mathrm{~nm})$ is consistent with the measured $J-V$ parameters. Moreover, we fabricated reference PSCs with spiro-OMeTAD as HTL to confirm not only the quality of the perovskite layer, but also of the other device layers, and the whole device architecture. The reference PSC when using spiro-OMeTAD as HTL yielded a PCE of $20.4 \%$, with a $\mathrm{V}_{\mathrm{OC}}=1169 \mathrm{mV}$ and $\mathrm{J}_{\mathrm{SC}}=22.6 \mathrm{~mA} \mathrm{~cm}^{-2}$ (Figure 1d). The performance difference between the spiro-OMeTAD-based and the PDPP-3T-based devices can be explained by different electronic properties of the HTL materials. For instance, PDPP-3T is a dopant-free HTL, while spiro-OMeTAD is typically doped with tBP and Li-TFSI in order to enhance both the hole mobility and conductivity, leading to improved device performance..$^{44-45}$ 
Table 1. Device performance and figures-of-merit of the champion PSCs prepared with different perovskite layer thicknesses. The scan rate was $50 \mathrm{mV} \mathrm{s}^{-1}$.

\begin{tabular}{|c|c|c|c|c|c|}
\hline $\begin{array}{l}\text { Perovskite } \\
\text { thickness }\end{array}$ & $\begin{array}{c}\text { Scan } \\
\text { direction }\end{array}$ & $\begin{array}{c}\mathrm{J}_{\mathrm{SC}} \\
\left(\mathrm{mA} \mathbf{c m}^{-2}\right)\end{array}$ & $\begin{array}{l}\text { VoC } \\
(\mathrm{mV})\end{array}$ & $\begin{array}{l}\text { FF } \\
(\%)\end{array}$ & $\begin{array}{c}\eta \\
(\%)\end{array}$ \\
\hline \multirow{2}{*}{$170 \mathrm{~nm}$} & $J_{S C}$ to $V_{O C}$ & 17.5 & 1097 & 64.9 & 12.5 \\
\hline & $V_{O C}$ to $J_{S C}$ & 17.2 & 1088 & 64.3 & 12.0 \\
\hline \multirow{2}{*}{$360 \mathrm{~nm}$} & $J_{S C}$ to $V_{O C}$ & 18.4 & 1100 & 68.9 & 13.4 \\
\hline & $V_{O C}$ to $J_{S C}$ & 18.5 & 1100 & 67.3 & 13.7 \\
\hline \multirow{2}{*}{$570 \mathrm{~nm}$} & $J_{S C}$ to $V_{O C}$ & 19.7 & 1133 & 72.3 & 16.1 \\
\hline & $V_{O C}$ to $J_{S C}$ & 19.7 & 1133 & 70.4 & 15.7 \\
\hline
\end{tabular}

\section{ps-ns Charge Carrier Dynamics}

Charge extraction from the perovskite layer to the respective charge transport layers typically occurs on the sub-nanosecond timescale. ${ }^{15}$ Hence, TA measurements were conducted in the ps-ns time range to monitor the hole extraction process at the perovskite/PDPP-3T interface following optical excitation of the perovskite layer at $600 \mathrm{~nm}$. The respective TA spectra are presented in Figure 2. The spectra exhibit the characteristic GSB of the perovskite around $1.60 \mathrm{eV}$, accompanied by a broad GSB in the spectral region from $1.40-1.50 \mathrm{eV}$, which we assigned to the GSB of the PDPP-3T used as HTL. To compare, Figure S8 shows the TA spectra of perovskite/spiro-OMeTAD samples. They are identical to those of neat perovskite films. The assignment to the GSB of the PDPP-3T polymer is supported by comparison to the TA spectra of neat PDPP-3T films shown in Figure 2a. The respective kinetics of the neat PDPP-3T film GSB extracted in the spectral region of $1.40-1.50 \mathrm{eV}$ are displayed in Figure S9. The full ground state recovery in neat PDPP-3T films was determined to occur within 45 ps. The total signal intensity 
of the perovskite GSB was found to be higher when exciting from the perovskite side compared to excitation from the HTL side, both in the picosecond-nanosecond (ps-ns) and nanosecond-microsecond (ns- $-\mu$ s) time range (Figure S11 and Figure S13). We hypothesize that this is due to the filter effect of the PDPP-3T layer, which absorbs a fraction of the incident excitation light.

(a)

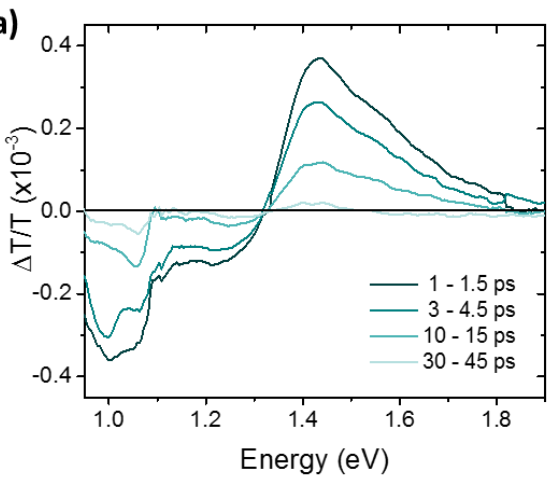

(b)

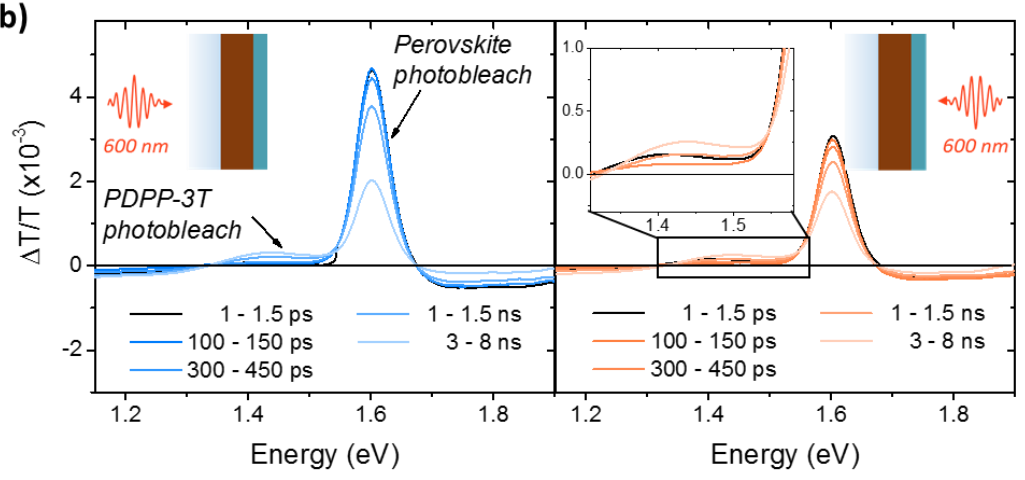

Figure 2. The $\mathrm{ps}^{-} \mathrm{ns}$ transient absorption spectra of (a) neat PDPP-3T polymer film, and (b) perovskite (170 nm)/PDPP-3T sample excited from the perovskite side (left) and HTL side (right). The inset (right) shows a magnification of the PDPP-3T GSB region. The samples were excited at $600 \mathrm{~nm}$ using a laser fluence of $0.37 \mu \mathrm{J} \mathrm{cm}^{-2}$. The inset schemes in (b) indicate the incidence of the optical excitation pulse on the samples.

In fact, photoexcitation of the perovskite/PDPP-3T samples not only created charges in the perovskite, but also excitons in the polymeric HTL, yet to a lesser extent when the excitation was incident on the perovskite side due to the high absorbance of the perovskite layer. Moreover, in the perovskite/HTL bilayer samples, the PDPP-3T GSB first decayed entirely within tens of ps, while the perovskite GSB remained, followed by an increase of the HTL GSB, accompanied by a decay of the perovskite GSB. This clearly indicates that first photogenerated excitons on PDPP3T decayed and then extraction of holes from the perovskite layer to the PDPP-3T HTL (Figure 
3) occurred. In the case of excitation from the perovskite side, we cannot exclude that trapping of electrons occurred in parallel to hole extraction and electron-hole recombination; electron trapping in deep sub-bandgap states has been shown to lead to fast non-radiative recombination. ${ }^{28}$

Irrespective of the excitation direction, we observed that the perovskite GSB started decaying after 100-150 ps, as shown in Figure 3 and Figure S11-S12, indicating the onset of carrier extraction to the HTL. Excitation from the HTL side resulted in an overall slower decay of the perovskite GSB, which became more prominent in thicker samples (Figure S12). Precisely, following excitation from the HTL side, $80 \%$ of the photogenerated carriers (electrons and holes) remained in the $570 \mathrm{~nm}$-thick perovskite layer after $8 \mathrm{~ns}$, while for the $360 \mathrm{~nm}$ and $170 \mathrm{~nm}$-thick perovskite/PDPP-3T samples only $69 \%$ and $48 \%$, respectively, of the photogenerated charge carriers were still present after $8 \mathrm{~ns}$. The improved crystal quality of the perovskite film in thicker layers can reduce bulk and interface recombination, since with the film quality also the interface quality of the perovskite/PDPP-3T junction improves. ${ }^{43,46}$

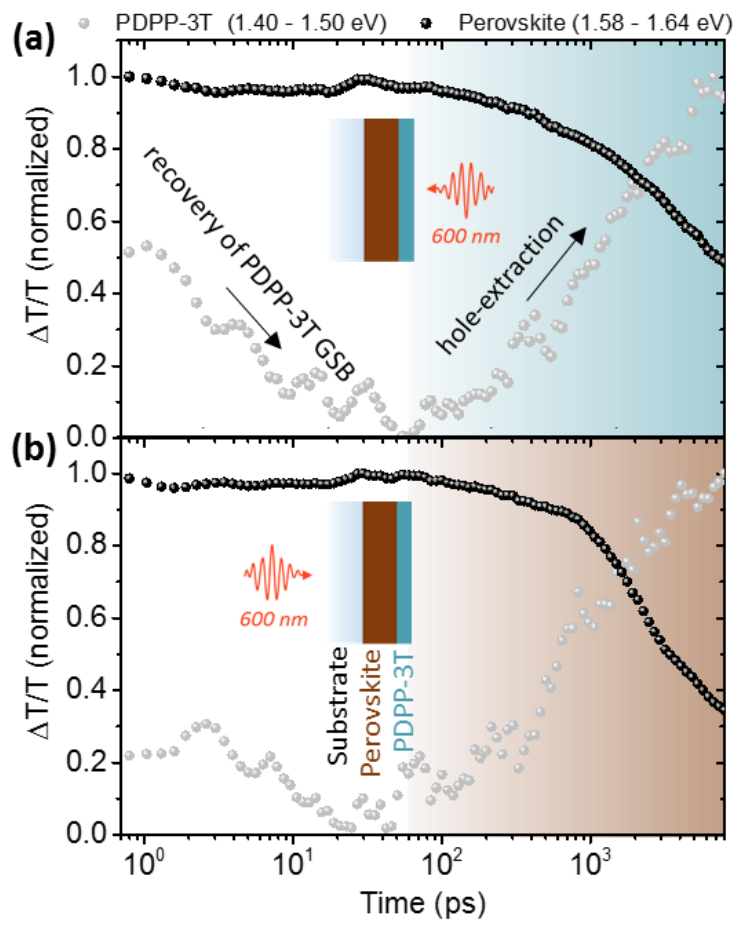


Figure 3. Normalized $\mathrm{ps}^{-} \mathrm{ns}$ charge carrier dynamics of semi-device-like perovskite (170 $\mathrm{nm}$ )/PDPP-3T sample integrated from 1.40-1.50 eV (PDPP-3T GSB) and 1.58-1.64 eV (perovskite GSB) following excitation from the (a) HTL side and (b) the perovskite side. The samples were excited at $600 \mathrm{~nm}$ using a laser fluence of $0.37 \mu \mathrm{J} \mathrm{cm}^{-2}$. Insets show the incidence of the excitation. Note that the initial fast GSB recovery of PDPP-3T is due to direct photoexcitation of the polymer, creating short-lived singlet excitons.

\section{ns- - s Charge Carrier Dynamics}

To further evaluate the charge carrier recombination dynamics beyond the ps-ns timescale, we probed the PDPP-3T GSB also in the ns- $\mu$ s time range (Figure S13 and Figure S14). In general, charge carrier motion in the absorber layer (i.e., the perovskite film) occurs in devices via both drift and diffusion. ${ }^{47-48}$ Here, we varied the thickness of the perovskite films in the perovskite/PDPP-3T samples to study the carrier diffusion process in the absorber layer without any external bias, that is, in the absence of drift. In this case, the rise time of the PDPP-3T GSB is expected to depend on the incidence of excitation as in the case of excitation from the perovskite side, carriers first have to diffuse through the perovskite layer before they reach the HTL interface, where they extraction takes place. Figure 4 shows the charge carrier dynamics in the PDPP-3T monitored across the probe region from $1.40-1.50 \mathrm{eV}$, the region of the polymer's GSB, for both excitation incidences. Clearly, the PDPP-3T GSB signal rises in the first $10 \mathrm{~ns}$, when exciting from the HTL side. The rise time is independent of the perovskite film thickness, since most of the charge carriers were generated closely to the perovskite/PDPP-3T interface, when exciting from the HTL side (Table S1). 


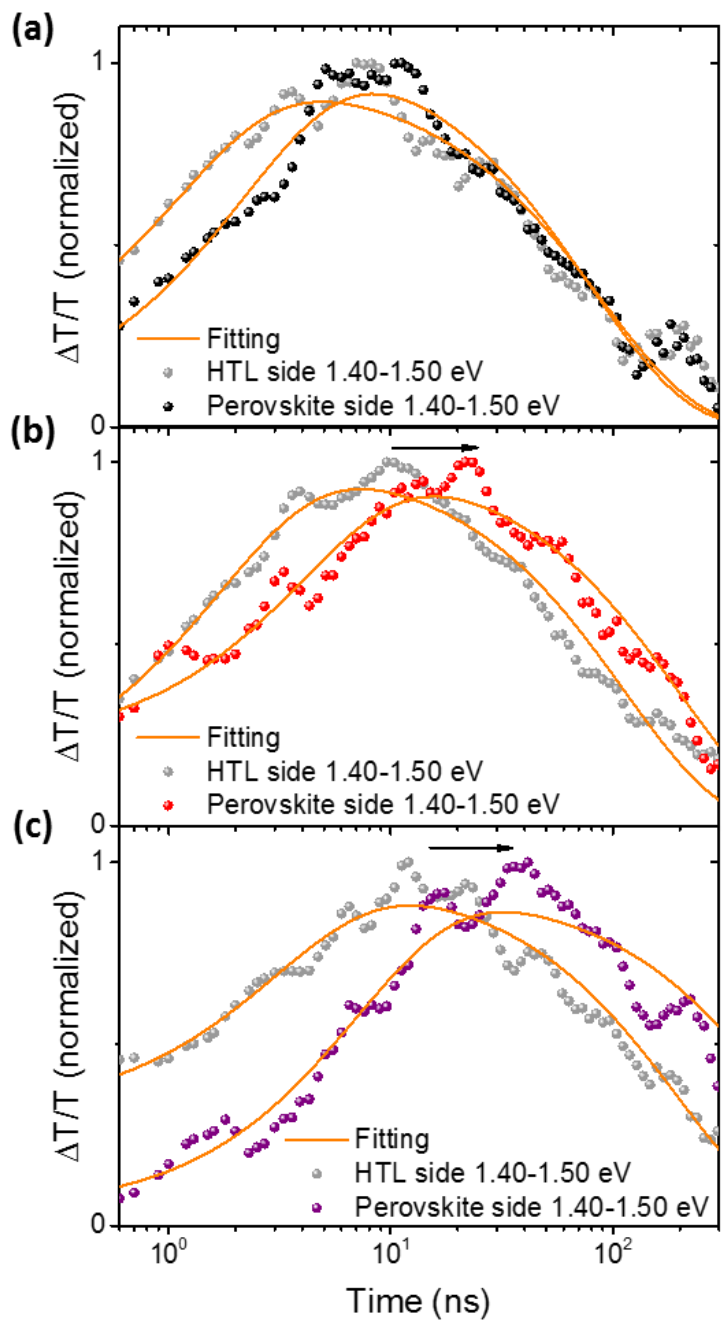

Figure 4. Normalized ns $-\mu$ s charge carrier dynamics monitored at the spectral position of the PDPP-3T GSB (1.40-1.50 eV) for different excitation incidences on the perovskite/PDPP-3T samples following excitation at $532 \mathrm{~nm}$ of (a) $170 \mathrm{~nm}$, (b) $360 \mathrm{~nm}$, and (c) $570 \mathrm{~nm}$-thick perovskite absorber layers. The dots represent the experimental data, while the solid lines are fits to the data using the sum of an exponential rise and exponential decay function. The arrows indicate the shift of the peak time of the PDPP-3T GSB following excitation from the perovskite side.

On the other hand, in the case of excitation from the perovskite side, the hole extraction to the PDPP-3T depends on the diffusion of holes through the absorber layer to the interface with the HTL. Only, in the case of the $170 \mathrm{~nm}$-thick perovskite sample, the dynamics of the PDPP-3T GSB 
is identical regardless of the incidence of the excitation (Figure 4a). This implies that charge carriers either are generated throughout the entire perovskite layer thickness (due to the penetration depth of the excitation pulse, as shown in Table S1) and / or diffuse very fast, which prevents determination of a clear transit time of charges through the perovskite layer to the perovskite/PDPP-3T interface. Figures 4b-c display the 'delay' of the hole extraction that occurs with increasing perovskite film thickness, following excitation of the sample incident on the perovskite side. Under these conditions, charge carriers have to diffuse to the HTL prior to extraction (Figure S15). We deduced the hole extraction rate $\left(k_{h}\right)$ at the perovskite/PDPP-3T interface by fitting an exponential rise to the data. The extracted $k_{h}$ for different layer thickness are comparable when the excitation was incident on the HTL side (Table 2), indicating that we observe the rate of the interfacial hole transfer process. Clearly, when incident on the perovskite side, the 'effective' hole extraction rate decreased as the thickness of the perovskite absorber layer increased, due to the need for carriers to diffuse through the perovskite film prior to extraction as depicted in Figure 4 and Table 2.

Table 2. Hole extraction rates for perovskite/PDPP-3T samples extracted from the PDPP-3T GSB dynamics after excitation of the perovskite from different directions.

\begin{tabular}{ccccc} 
Perovskite layer thickness & $\mathbf{1 7 0} \mathbf{~ n m}$ & $\mathbf{3 6 0} \mathbf{~ n m}$ & $\mathbf{5 7 0} \mathbf{~ n m}$ \\
\hline & $\begin{array}{c}\text { Excitation incident on } \\
\text { perovskite side }\end{array}$ & $4.5 \times 10^{8}$ & $3.1 \times 10^{8}$ & $1.2 \times 10^{8}$ \\
\cline { 2 - 4 } $\boldsymbol{k}_{\boldsymbol{h}}\left(\mathbf{s}^{\mathbf{- 1}}\right)$ & $\begin{array}{c}\text { Excitation incident on } \\
\text { HTL side }\end{array}$ & $6.6 \times 10^{8}$ & $6.3 \times 10^{8}$ & $4.5 \times 10^{8}$ \\
\hline
\end{tabular}


In order to account for the carrier diffusion process and to estimate the charge carrier mobility, we applied a simple one-dimensional diffusion model. We tracked the perovskite GSB of the perovskite/PDPP-3T sample (Figure S14), and simulated the charge carrier dynamics (further details are given in Supporting Information). Here, we used the data of the perovskite sample with $\mathrm{d} \sim 170 \mathrm{~nm}$ excited at a fluence of $\sim 0.4 \mu \mathrm{J} / \mathrm{cm}^{2}$ (Figure S17) for the calculation, since the carrier dynamics is fluence-independent at low excitation density. The fitting results are shown in Table S2. Furthermore, we estimated the 'effective' hole extraction rate from the perovskite GSB using the following equation ${ }^{49}$ :

$$
k_{h, P V K} \sim \frac{1}{\frac{d}{S_{h}}+\frac{4}{D}\left(\frac{d}{\pi}\right)^{2}}
$$

The equation above indicates that $k_{h, P V K}$ decreases with the sample thickness, which is consistent with our experimental observation (Table 2 and Table S3). However, the change in the measured $k_{h, P V K}$ associated with the excitation direction is not inferred from this equation. To illustrate the discrepancy, we simulated the rising edge of the hole injection to the PDPP-3T HTL. The time evolution of the hole densities at the perovskite/PDPP-3T interface are shown for each sample in Figure S16. We confirmed that the hole density requires time to build-up, when exciting from the perovskite side as a consequence of carrier diffusion. The build-up time increases with perovskite layer thickness, which alters the 'effective' hole extraction rates.

Next, we calculated the carrier mobility $\mu$ and the diffusion length $L$ by the following relations ${ }^{5}$, 50.

$$
\mu=\frac{D q}{k_{B} T}, \quad L=\sqrt{D \tau_{b}}
$$


where, $k_{B} T$ is the product of the Boltzmann constant and the temperature (equivalent to $25.7 \mathrm{meV}$ at room temperature), $q$ is the electrical charge. Thereby, we approximated the carrier mobility to be around $39 \mathrm{~cm}^{2} \mathrm{~V}^{-1} \mathrm{~s}^{-1}$ and the diffusion length to be $3.79 \mu \mathrm{m}$ using $\tau_{b}=145 \mathrm{~ns}$, determined from a single exponential fit to the GSB recovery of the $170 \mathrm{~nm}$-thick perovskite film at low excitation fluence. We note that the mobility values we obtained are consistent with those reported elsewhere for mixed-halide perovskites. ${ }^{51-53}$ The high hole mobility can be explained by high crystallinity of the absorber layer, since the presence of structural imperfections causes energetic disorder, which limits the charge carrier mobility in metal halide perovskites. ${ }^{50}$

To further investigate the carrier dynamics at the perovskite/PDPP-3T interface, we also performed fluence-dependent measurements on the ns $-\mu$ s timescale. Here, we used the $170 \mathrm{~nm}-$ thick neat perovskite and the perovskite $(170 \mathrm{~nm}) / \mathrm{PDPP}-3 \mathrm{~T}$ sample as shown in Figure 5, since for this sample the charge carrier dynamics were found to be independent of the incidence of the excitation. When the fluence was increased from 0.2 to $3.2 \mu \mathrm{J} \mathrm{cm}^{-2}$, the perovskite GSB dynamics of both neat perovskite and perovskite/PDPP-3T samples showed faster decay (Figure S17). In Figure 5c, we show the charge carrier dynamics in PDPP-3T to investigate fluence dependent hole extraction. Independent of the excitation fluence, the hole extraction process concluded within 10 ns (Figure 5c and Figure S17), and only 50\% of the holes remained after 30 ns. Interestingly, the rise time of the signal intensity of the PDPP-3T GSB continuously increased with the excitation fluence up to $0.8 \mu \mathrm{J} \mathrm{cm}^{-2}$ (Figure 5c). At this point, the peak intensity of the PDPP-3T GSB reached a maximum, which indicates that the hole extraction to the PDPP-3T layer could be limited by the build-up of space charge at the perovskite/PDPP-3T interface and high carrier concentration in the PDPP-3T polymer layer. ${ }^{47,}{ }^{54}$ Moreover, the GSB of PDPP-3T displayed fluenceindependent decay for fluences higher than $0.8 \mu \mathrm{J} \mathrm{cm}^{-2}$ (Figure S18). However, we note that the 
device performance cannot be directly correlated to the recombination of holes at the perovskite/PDPP-3T after 10 ns as shown in Figure 5c and Figure S17, since the holes extracted at the perovskite/PDPP-3T interface naturally remained in the HTL and were not extracted as they would have been in a complete device structure. Therefore, after $10 \mathrm{~ns}$ carrier recombination at the perovskite/PDPP-3T interface dominates, and a recombination rate of $k_{\text {hole, recomb }}=1.8 \times 10^{7} \mathrm{~s}^{-1}$ (Figure 5d) was determined.
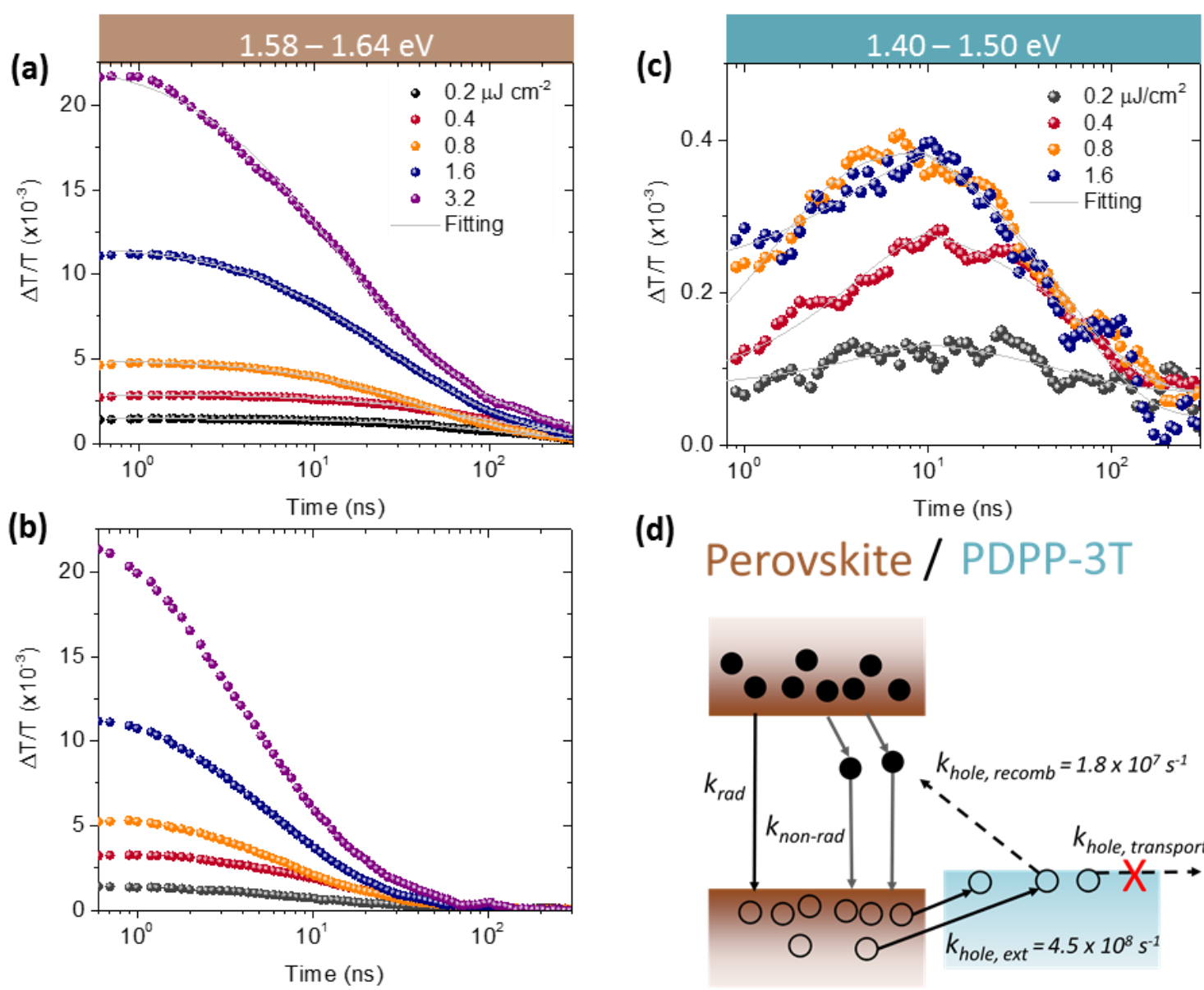

(d)

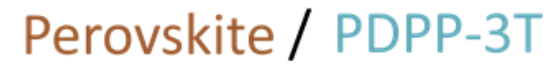


of the intrinsic carrier recombination $\left(k_{\text {rad }}, k_{\text {non-rad }}\right)$, hole extraction $\left(k_{\text {hole,ext }}\right)$, and interface recombination $\left(k_{\text {hole,recomb }}\right)$ processes in the perovskite/PDPP-3T samples.

Finally, we performed ns- $\mu$ s TA measurements also on $\mathrm{TiO}_{2} /$ perovskite/PDPP-3T stacks that mimic the entire device structure (here $\mathrm{TiO}_{2}$ denotes c- $\mathrm{TiO}_{2} / \mathrm{m}-\mathrm{TiO}_{2}$ layers with PMMA:PCBM passivation). The device-like ETL/perovskite/HTL samples provide additional insight to the recombination of carriers in the presence of both electron and hole extraction layers. The $\mathrm{TiO}_{2} /$ perovskite/PDPP-3T stacks exhibited slower perovskite ground state recovery at later time points, as shown in Figure S20. In case of excitation incident on the $\mathrm{TiO}_{2}$ side of the $\mathrm{TiO}_{2} /$ perovskite $(570 \mathrm{~nm}) / \mathrm{PDPP}-3 \mathrm{~T}$ stack, $25 \%$ of the perovskite GSB signal remained after 100 ns. In contrast, only $\sim 10 \%$ of the TA signal remained in the case of the perovskite/PDPP-3T sample without the ETL (Figure S14 and Figure S20). At earlier delay times, i.e. within the first $10 \mathrm{~ns}$, the perovskite GSB of the $\mathrm{TiO}_{2} /$ perovskite $(570 \mathrm{~nm}) / \mathrm{PDPP}-3 \mathrm{~T}$ sample decayed slightly faster compared to the perovskite/PDPP-3T sample. Thus, it appears that the extraction of both holes and electrons from the perovskite layer results in a reduced charge carrier concentration in the perovskite film and thereby slower recombination. ${ }^{30}$ Recently, it has been shown that using PMMA:PCBM as passivation layer between $\mathrm{TiO}_{2}$ and perovskite further reduces the interfacial recombination and in turn increases the lifetime of photogenerated carriers. ${ }^{13,29,46,55}$ Additionally, we observed a pronounced spectral shift of the perovskite GSB when the perovskite layer thickness was increased and when the film was excited incident on the HTL side (Figure S19). In contrast, no spectral shift of the perovskite GSB band was observed, when the optical excitation was performed incident on the ETL side. The blue-shift of spectra is evidence of a reduced trap density due to improved crystal quality of the perovskite layer. ${ }^{56-57}$ Hence, it appears that the crystallization 
of the perovskite is not entirely uniform across the perovskite absorber layer, when deposited on $\mathrm{m}-\mathrm{TiO}_{2}$.

To conclude, PDPP-3T has been successfully implemented as HTL in PSCs. TA spectroscopy measurements were carried out to probe the charge carrier extraction mechanism and dynamics at the perovskite/HTL interface. Due to the lower energy ground state absorption of the polymer PDPP-3T with respect to that of the perovskite layer, direct observation of hole extraction from the perovskite to the PDPP-3T was possible by probing the ground state bleach of the HTL. The hole extraction times were determined to be on the order of $10 \mathrm{~ns}$. Furthermore, measurements on perovskite thin films with different film thickness revealed a delayed rise of the PDPP-3T GSB, indicating carrier diffusion through the perovskite absorber layer. The diffusion coefficient was obtained by fitting the perovskite/PDPP-3T carrier dynamics using a one-dimensional diffusion equation and with the diffusion coefficient in hand, the perovskite's carrier mobility was determined. Finally, TA spectroscopy measurements indicated that the distribution of trap states across the perovskite absorber layer was not uniform, when a mesoporous scaffold was used as the charge transport layer. 


\section{ASSOCIATED CONTENT}

Supporting Information: Experimental details, supplementary characterizations of materials, device fabrication, and TA spectroscopy experimental details with analysis.

\section{AUTHOR INFORMATION \\ Corresponding Authors \\ *e-mail: frederic.laquai@kaust.edu.sa}

\section{Web page:}

Ultrafast Dynamics Group: https://ufd.kaust.edu.sa

\section{Orcid:}

Esma Ugur: 0000-0003-0070-334X

Jafar I. Khan: 0000-0001-6003-5641

Erkan Aydin: 0000-0002-8849-2788

Iain McCulloch: 0000-0002-6340-7217

Stefaan De Wolf: 0000-0003-1619-9061

Frédéric Laquai: $\underline{0000-0002-5887-6158}$

\section{Notes}

E.U and J.I.K equally contributed to this work.

The authors declare no competing financial interest.

ACKNOWLEDGEMENT 
This publication is based upon work supported by the King Abdullah University of Science and Technology (KAUST) Office of Sponsored Research (OSR) under Award No: OSR-2018CARF/CCF-3079. 


\section{REFERENCES}

(1) NREL, Best Research-Cell Efficiency Chart. 2019, https://www.nrel.gov/pv/assets/pdfs/ best-research-cell-efficiencies.20190802.pdf(accessed: August 5, 2019).

(2) Lin, Q.; Armin, A.; Nagiri, R. C. R.; Burn, P. L.; Meredith, P. Electro-optics of Perovskite Solar Cells. Nat. Photonics 2014, 9, 106-112.

(3) Wang, Y.; Zhang, Y.; Zhang, P.; Zhang, W. High Intrinsic Carrier Mobility and Photon Absorption in the Perovskite $\mathrm{CH}_{3} \mathrm{NH}_{3} \mathrm{PbI}_{3}$. Phys. Chem. Chem. Phys. 2015, 17, 11516-11520.

(4) De Wolf, S.; Holovsky, J.; Moon, S. J.; Loper, P.; Niesen, B.; Ledinsky, M.; Haug, F. J.; Yum, J. H.; Ballif, C. Organometallic Halide Perovskites: Sharp Optical Absorption Edge and Its Relation to Photovoltaic Performance. J. Phys. Chem. Lett. 2014, 5, 1035-1039.

(5) Stranks, S. D.; Eperon, G. E.; Grancini, G.; Menelaou, C.; Alcocer, M. J. P.; Leijtens, T.; Herz, L. M.; Petrozza, A.; Henry J. Snaith. Electron-Hole Diffusion Lengths Exceeding 1 Micrometer in an Organometal Trihalide Perovskite Absorber. Science 2013, 342, 341-344.

(6) Xing, G.; Mathews, N.; Sun, S.; Lim, S. S.; Lam, Y. M.; Grätzel, M.; Mhaisalkar, S.; Sum, T. C. Long-Range Balanced Electron- and Hole-Transport Lengths in Organic-Inorganic $\mathrm{CH}_{3} \mathrm{NH}_{3} \mathrm{PbI}_{3}$. Science 2013, 342, 344-347.

(7) Steirer, K. X.; Schulz, P.; Teeter, G.; Stevanovic, V.; Yang, M.; Zhu, K.; Berry, J. J. Defect Tolerance in Methylammonium Lead Triiodide Perovskite. ACS Energy Lett. 2016, 1, $360-366$.

(8) Meggiolaro, D.; Motti, S. G.; Mosconi, E.; Barker, A. J.; Ball, J.; Andrea Riccardo Perini, C.; Deschler, F.; Petrozza, A.; De Angelis, F. Iodine Chemistry Determines the Defect Tolerance of Lead-halide Perovskites. Energy Environ. Sci. 2018, 11, 702-713. 
(9) Ledinsky, M.; Schönfeldová, T.; Holovský, J.; Aydin, E.; Hájková, Z.; Landová, L.;

Neyková, N.; Fejfar, A.; De Wolf, S. Temperature Dependence of the Urbach Energy in Lead Iodide Perovskites. J. Phys. Chem. Lett. 2019, 10, 1368-1373.

(10) Würfel, U.; Cuevas, A.; Würfel, P. Charge Carrier Separation in Solar Cells. IEEE J. Photovoltaics 2015, 5, 461-469.

(11) Ravishankar, S.; Gharibzadeh, S.; Roldán-Carmona, C.; Grancini, G.; Lee, Y.; Ralaiarisoa, M.; Asiri, A. M.; Koch, N.; Bisquert, J.; Nazeeruddin, M. K. Influence of Charge Transport Layers on Open-Circuit Voltage and Hysteresis in Perovskite Solar Cells. Joule 2018, 2, 788-798.

(12) Aydin, E.; Troughton, J.; De Bastiani, M.; Ugur, E.; Sajjad, M.; Alzahrani, A.;

Neophytou, M.; Schwingenschlögl, U.; Laquai, F.; Baran, D.; De Wolf, S. Room-TemperatureSputtered Nanocrystalline Nickel Oxide as Hole Transport Layer for $\mathrm{p}-\mathrm{i}-\mathrm{n}$ Perovskite Solar Cells. ACS Appl. Energy Mater. 2018, 1, 6227-6233.

(13) Peng, J.; Khan, J. I.; Liu, W.; Ugur, E.; Duong, T.; Wu, Y.; Shen, H.; Wang, K.; Dang, H.; Aydin, E., et al. A Universal Double-Side Passivation for High Open-Circuit Voltage in Perovskite Solar Cells: Role of Carbonyl Groups in Poly(methyl methacrylate). Adv. Energy Mater. 2018, 8, 1801208.

(14) Aydin, E.; De Bastiani, M.; De Wolf, S. Defect and Contact Passivation for Perovskite Solar Cells. Adv. Mater. 2019, 31, 1900428.

(15) Shi, J.; Li, Y.; Li, Y.; Li, D.; Luo, Y.; Wu, H.; Meng, Q. From Ultrafast to Ultraslow: Charge-Carrier Dynamics of Perovskite Solar Cells. Joule 2018, 2, 879-901. 
(16) Jiang, Q.; Zhang, L.; Wang, H.; Yang, X.; Meng, J.; Liu, H.; Yin, Z.; Wu, J.; Zhang, X.;

You, J. Enhanced Electron Extraction Using $\mathrm{SnO}_{2}$ for High-Efficiency Planar-Structure

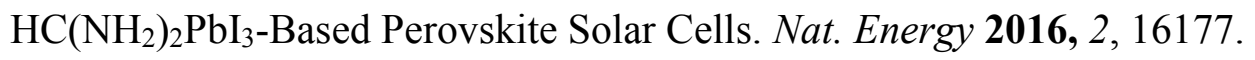

(17) Heo, J. H.; Park, S.; Im, S. H.; Son, H. J. Development of Dopant-Free Donor-Acceptortype Hole Transporting Material for Highly Efficient and Stable Perovskite Solar Cells. ACS Appl. Mater. Interfaces 2017, 9, 39511-39518.

(18) Yongsheng, L.; Ziruo, H.; Qi, C.; Huajun, C.; Wei-Hsuan, C.; Yang, Y.; Tze-Bin, S.; Yang, Y. Perovskite Solar Cells Employing Dopant-Free Organic Hole Transport Materials with Tunable Energy Levels. Adv. Mater. 2016, 28, 440-446.

$$
\text { Li, Z. A.; Zhu, Z.; Chueh, C.-C.; Jo, S. B.; Luo, J.; Jang, S.-H.; Jen, A. K. Y. Rational }
$$

Design of Dipolar Chromophore as an Efficient Dopant-Free Hole-Transporting Material for Perovskite Solar Cells. J. Am. Chem. Soc. 2016, 138, 11833-11839.

(20) Chiang, Y.-H.; Chou, H.-H.; Cheng, W.-T.; Li, Y.-R.; Yeh, C.-Y.; Chen, P. Porphyrin Dimers as Hole-Transporting Layers for High-Efficiency and Stable Perovskite Solar Cells. ACS Energy Lett. 2018, 3, 1620-1626.

(21) Dubey, A.; Adhikari, N.; Venkatesan, S.; Gu, S.; Khatiwada, D.; Wang, Q.; Mohammad, L.; Kumar, M.; Qiao, Q. Solution Processed Pristine PDPP3T Polymer as Hole Transport Layer for Efficient Perovskite Solar Cells with Slower Degradation. Sol. Energy Mater. Sol. Cells 2016, 145, 193-199.

(22) Luo, J.; Xia, J.; Yang, H.; Chen, L.; Wan, Z.; Han, F.; Malik, H. A.; Zhu, X.; Jia, C. Toward High-Efficiency, Hysteresis-Less, Stable Perovskite Solar Cells: Unusual Doping of a Hole-Transporting Material Using a Fluorine-Containing Hydrophobic Lewis Acid. Energy Environ. Sci. 2018, 11, 2035-2045. 
(23) Kim, Y.; Jung, E. H.; Kim, G.; Kim, D.; Kim, B. J.; Seo, J. Sequentially Fluorinated PTAA Polymers for Enhancing Voc of High-Performance Perovskite Solar Cells. Adv. Energy Mater. 2018, 8, 1801668.

(24) Saliba, M.; Orlandi, S.; Matsui, T.; Aghazada, S.; Cavazzini, M.; Correa-Baena, J.-P.; Gao, P.; Scopelliti, R.; Mosconi, E.; Dahmen, K.-H., et al. A Molecularly Engineered HoleTransporting Material for Efficient Perovskite Solar Cells. Nat. Energy 2016, 1, 15017.

(25) Lamberti, F.; Gatti, T.; Cescon, E.; Sorrentino, R.; Rizzo, A.; Menna, E.; Meneghesso, G.; Meneghetti, M.; Petrozza, A.; Franco, L. Evidence of Spiro-OMeTAD De-Doping by tertButylpyridine Additive in Hole-Transporting Layers for Perovskite Solar Cells. Chem. 2019, 5, 1806-1817.

(26) Zhou, W.; Wen, Z.; Gao, P. Less is More: Dopant-Free Hole Transporting Materials for High-Efficiency Perovskite Solar Cells. Adv. Energy Mater. 2018, 8, 1702512.

(27) Hutter, E. M.; Hofman, J.-J.; Petrus, M. L.; Moes, M.; Abellón, R. D.; Docampo, P.; Savenije, T. J. Charge Transfer from Methylammonium Lead Iodide Perovskite to Organic Transport Materials: Efficiencies, Transfer Rates, and Interfacial Recombination. Adv. Energy Mater. 2017, 7, 1602349.

(28) Leijtens, T.; Eperon, G. E.; Barker, A. J.; Grancini, G.; Zhang, W.; Ball, J. M.; Kandada, A. R. S.; Snaith, H. J.; Petrozza, A. Carrier Trapping and Recombination: The Role of Defect Physics in Enhancing The Open Circuit Voltage of Metal Halide Perovskite Solar Cells. Energy Environ. Sci. 2016, 9, 3472-3481.

(29) Stolterfoht, M.; Wolff, C. M.; Márquez, J. A.; Zhang, S.; Hages, C. J.; Rothhardt, D.; Albrecht, S.; Burn, P. L.; Meredith, P.; Unold, T. et al. Visualization and Suppression of 
Interfacial Recombination for High-Efficiency Large-Area pin Perovskite Solar Cells. Nat. Energy 2018, 3, 847-854.

(30) Ihly, R.; Dowgiallo, A.-M.; Yang, M.; Schulz, P.; Stanton, N. J.; Reid, O. G.; Ferguson, A. J.; Zhu, K.; Berry, J. J.; Blackburn, J. L. Efficient Charge Extraction and Slow Recombination in Organic-Inorganic Perovskites Capped with Semiconducting Single-Walled Carbon Nanotubes. Energy Environ. Sci. 2016, 9, 1439-1449.

(31) Ishioka, K.; Barker, B. G.; Yanagida, M.; Shirai, Y.; Miyano, K. Direct Observation of Ultrafast Hole Injection from Lead Halide Perovskite by Differential Transient Transmission Spectroscopy. J. Phys. Chem. Lett. 2017, 8, 3902-3907.

(32) Serpetzoglou, E.; Konidakis, I.; Kakavelakis, G.; Maksudov, T.; Kymakis, E.; Stratakis, E. Improved Carrier Transport in Perovskite Solar Cells Probed by Femtosecond Transient Absorption Spectroscopy. ACS Appl. Mater. Interfaces 2017, 9, 43910-43919.

(33) Ponseca, C. S.; Hutter, E. M.; Piatkowski, P.; Cohen, B.; Pascher, T.; Douhal, A.;

Yartsev, A.; Sundström, V.; Savenije, T. J. Mechanism of Charge Transfer and Recombination Dynamics in Organo Metal Halide Perovskites and Organic Electrodes, PCBM, and SpiroOMeTAD: Role of Dark Carriers. J. Am. Chem. Soc. 2015, 137, 16043-16048.

(34) Brauer, J. C.; Lee, Y. H.; Nazeeruddin, M. K.; Banerji, N. Ultrafast Charge Carrier Dynamics in $\mathrm{CH}_{3} \mathrm{NH}_{3} \mathrm{PbI}_{3}$ : Evidence for Hot Hole Injection into Spiro-OMeTAD. J. Mater. Chem. C 2016, 4, 5922-5931.

(35) Leng, J.; Liu, J.; Zhang, J.; Jin, S. Decoupling Interfacial Charge Transfer from Bulk Diffusion Unravels Its Intrinsic Role for Efficient Charge Extraction in Perovskite Solar Cells. $J$. Phys. Chem. Lett. 2016, 7, 5056-5061. 
Corani, A.; Li, M.-H.; Shen, P.-S.; Chen, P.; Guo, T.-F.; El Nahhas, A.; Zheng, K.;

Yartsev, A.; Sundström, V.; Ponseca, C. S. Ultrafast Dynamics of Hole Injection and

Recombination in Organometal Halide Perovskite Using Nickel Oxide as p-Type Contact

Electrode. J. Phys. Chem. Lett. 2016, 7, 1096-1101.

(37) Brauer, J. C.; Lee, Y. H.; Nazeeruddin, M. K.; Banerji, N. Charge Transfer Dynamics

from Organometal Halide Perovskite to Polymeric Hole Transport Materials in Hybrid Solar Cells. J. Phys. Chem. Lett. 2015, 6, 3675-3681.

(38) Dang, H. X.; Wang, K.; Ghasemi, M.; Tang, M.-C.; De Bastiani, M.; Aydin, E.; Dauzon, E.; Barrit, D.; Peng, J.; Smilgies, D.-M., et al. A. Multi-cation Synergy Suppresses Phase Segregation in Mixed-Halide Perovskites. Joule 2019, 3, 1746-1764.

(39) Saliba, M.; Matsui, T.; Seo, J.-Y.; Domanski, K.; Correa-Baena, J.-P.; Nazeeruddin, M.

K.; Zakeeruddin, S. M.; Tress, W.; Abate, A.; Hagfeldt, A., et al. Cesium-containing Triple Cation Perovskite Solar Cells: Improved Stability, Reproducibility and High Efficiency. Energy Environ. Sci. 2016, 9, 1989-1997.

(40) Chen, Z.; Dong, Q.; Liu, Y.; Bao, C.; Fang, Y.; Lin, Y.; Tang, S.; Wang, Q.; Xiao, X.; Bai, Y., et al. Thin Single Crystal Perovskite Solar Cells to Harvest Below-bandgap Light Absorption. Nat. Commun. 2017, 8, 1890.

(41) Tress, W. Perovskite Solar Cells on the Way to Their Radiative Efficiency Limit Insights Into a Success Story of High Open-Circuit Voltage and Low Recombination. Adv. Energy Mater. 2017, 7, 1602358.

(42) Ugur, E.; Sheikh, A. D.; Munir, R.; Khan, J. I.; Barrit, D.; Amassian, A.; Laquai, F. Improved Morphology and Efficiency of $n-i-p$ Planar Perovskite Solar Cells by Processing with Glycol Ether Additives. ACS Energy Lett. 2017, 2, 1960-1968. 
(43) Stranks, S. D. Nonradiative Losses in Metal Halide Perovskites. ACS Energy Lett. 2017, $2,1515-1525$

(44) Juarez-Perez, E. J.; Leyden, M. R.; Wang, S.; Ono, L. K.; Hawash, Z.; Qi, Y. Role of the Dopants on the Morphological and Transport Properties of Spiro-MeOTAD Hole Transport Layer. Chem. Mater. 2016, 28, 5702-5709.

(45) Pellaroque, A.; Noel, N. K.; Habisreutinger, S. N.; Zhang, Y.; Barlow, S.; Marder, S. R.; Snaith, H. J. Efficient and Stable Perovskite Solar Cells Using Molybdenum Tris(dithiolene)s as p-Dopants for Spiro-OMeTAD. ACS Energy Lett. 2017, 2, 2044-2050.

(46) Abdi-Jalebi, M.; Andaji-Garmaroudi, Z.; Cacovich, S.; Stavrakas, C.; Philippe, B.;

Richter, J. M.; Alsari, M.; Booker, E. P.; Hutter, E. M.; Pearson, A. J. et al. Maximizing and Stabilizing Luminescence from Halide Perovskites with Potassium Passivation. Nature 2018, $555,497-501$.

(47) De Bastiani, M.; Aydin, E.; Allen, T.; Walter, D.; Fell, A.; Peng, J.; Gasparini, N.; Troughton, J.; Baran, D.; Weber, K., et al. Interfacial Dynamics and Contact Passivation in Perovskite Solar Cells. Adv. Electron. Mater. 2019, 5, 1800500.

(48) Bisquert, J. Theory of the Impedance of Electron Diffusion and Recombination in a Thin Layer. J. Phys. Chem. B 2002, 106, 325-333.

(49) Sproul, A. B. Dimensionless Solution of The Equation Describing The Effect of Surface Recombination on Carrier Decay in Semiconductors. J. Appl. Phys. 1994, 76, 2851-2854. (50) Herz, L. M. Charge-Carrier Mobilities in Metal Halide Perovskites: Fundamental Mechanisms and Limits. ACS Energy Lett. 2017, 2, 1539-1548. 
(51) McMeekin, D. P.; Sadoughi, G.; Rehman, W.; Eperon, G. E.; Saliba, M.; Hörantner, M. T.; Haghighirad, A.; Sakai, N.; Korte, L.; Rech, B., et al. A Mixed-cation Lead Mixed-halide Perovskite Absorber for Tandem Solar Cells. Science 2016, 351, 151-155.

(52) Rehman, W.; McMeekin, D. P.; Patel, J. B.; Milot, R. L.; Johnston, M. B.; Snaith, H. J.;

Herz, L. M. Photovoltaic Mixed-cation Lead Mixed-halide Perovskites: Links Between Crystallinity, Photo-stability and Electronic Properties. Energy Environ. Sci. 2017, 10, 361-369. (53) McMeekin, D. P.; Wang, Z.; Rehman, W.; Pulvirenti, F.; Patel, J. B.; Noel, N. K.; Johnston, M. B.; Marder, S. R.; Herz, L. M.; Snaith, H. J. Crystallization Kinetics and Morphology Control of Formamidinium-Cesium Mixed-Cation Lead Mixed-Halide Perovskite via Tunability of the Colloidal Precursor Solution. Adv. Mater. 2017, 29, 1607039.

(54) Lopez-Varo, P.; Jiménez-Tejada, J. A.; García-Rosell, M.; Anta, J. A.; Ravishankar, S.; Bou, A.; Bisquert, J. Effects of Ion Distributions on Charge Collection in Perovskite Solar Cells. ACS Energy Lett. 2017, 2, 1450-1453

(55) Krogmeier, B.; Staub, F.; Grabowski, D.; Rau, U.; Kirchartz, T. Quantitative Analysis of The Transient Photoluminescence of $\mathrm{CH}_{3} \mathrm{NH}_{3} \mathrm{PbI}_{3} / \mathrm{PC}_{61} \mathrm{BM}$ Heterojunctions by Numerical Simulations. Sustainable Energy Fuels 2018, 2, 1027-1034.

(56) deQuilettes, D. W.; Vorpahl, S. M.; Stranks, S. D.; Nagaoka, H.; Eperon, G. E.; Ziffer, M. E.; Snaith, H. J.; Ginger, D. S. Impact of Microstructure on Local Carrier Lifetime in Perovskite Solar Cells. Science 2015, 348, 683-686.

(57) Mastroianni, S.; Heinz, F. D.; Im, J. H.; Veurman, W.; Padilla, M.; Schubert, M. C.; Würfel, U.; Grätzel, M.; Park, N. G.; Hinsch, A. Analysing the Effect of Crystal Size and Structure in Highly Efficient $\mathrm{CH}_{3} \mathrm{NH}_{3} \mathrm{PbI}_{3}$ Perovskite Solar Cells by Spatially Resolved Photo- and Electroluminescence Imaging. Nanoscale 2015, 7, 19653-19662. 